\title{
Latencies for movement naming with congruent and incongruent word stimuli
}

\author{
FREDERICK N. DYER* \\ U.S. Army Medical Research Laboratory, Fort Knox, Ky. 40121
}

Naming of movement directions was found to be subject to a small but significant amount of interference from incongruent names integrated with the movement stimuli. The delay of direction naming was less than the large delay of color naming when colors and incongruent color names were combined in the Stroop test. A hypothesis that faster processing of movement directions than of colors is the basis of this difference in interference was tested by beginning the processing of the words at various intervals prior to their movement. No appreciable increase in interference resulted from any of these stationary preexposures of the words. When word preexposures were longest (200 and $300 \mathrm{msec}$ ), interference was reduced. The reduced naming interference for movement direction and other dimensions compared to color suggests a basic difference between the central processing of color and other dimensions. Conditions with congruent combination of words and directions provided substantial speeding of direction naming over control conditions.

Color naming is delayed considerably when the patch is a color name denoting a color different from the color of the patch (Stroop, 1935; Jensen \& Rohwer, 1966). Shor (1970, 1971) has shown that naming of dimensions other than color can also be delayed by combination of a level of that dimension with the name of an incongruent dimension level (e.g., the word left written within an arrow pointing right). In practically all cases, naming of these other dimensions was less subject to interference than was the naming of colors. Despite their close similarity to color, even achromatic shades were found to be subject to less naming interference than was color (Dyer, 1971a).

Slowing word processing relative to color processing has been shown to reduce Stroop interference (Gumenik \& Glass, 1970). A possible explanation of the lower interference to naming for dimensions other than color is that the processing of these dimensions is faster than the processing of color. Because of this, the Gumenik and Glass condition of slow word processing relative to processing of the dimension to be named already exists for these other dimensions. Such an explanation appeared to account for the differences in interference between naming of colors and achromatic shades (Dyer, 1971a).

If processing of a stimulus dimension was too fast for words to interfere with naming of the level of the dimension, then speeding word processing relative to the processing of the dimension would be expected to increase the word's interference to

*The author wishes to thank Thomas $\mathbf{E}$ Kuehne, who collected the experimental data, and William J. Kelly, who produced the basic computer program. naming of the dimension. One way of speeding word processing would be to start such processing earlier than the processing of the dimension to be named. In the Stroop test, for example, a color word could be presented in a neutral color, such as black, for some period prior to its coloration (Dyer, 1971b). In the present study, words denoting the directions up, down, right, and left were moved in one of these directions, with the S's task being to name the direction of movement. One of the major independent variables of the study was the duration of a stationary exposure of the word prior to its movement. Pilot work had indicated that without such preexposures, i.e., when the word moved as soon as it was viewed, there was only a small delay in naming of the movement when the stimulus was a word denoting a movement direction incongruent to the actual movement direction compared to naming the direction of moving $\mathrm{Xs}_{\mathrm{s}}$. It was expected that some stationary preexposure of the word would produce a central interaction of the processed word and movement direction such that naming of the movement direction would be interfered with to the extent that color naming could be interfered with by a combination of an incongruent word with the color stimulus. The method of presentation of stimuli followed that of Dalrymple-Alford and Budayr (1966), who showed that individual color-word stimuli as well as cards with a series of such stimuli produced the Stroop phenomenon. Following Sichel and Chandler (1969), who used a similar procedure, congruent as well as incongruent stimulus combinations were included in the sequences of the present studies.

\section{EXPERIMENT 1 Method}

Subjects. Eight males, who ranged from 17 to 22 years of age and who had recently completed Army basic training, served as Ss. Visual acuity of both eyes was within normal Orthorater limits.

Apparatus and stimuli. Stimuli consisted of the words up, down, right, and left plus a series of four $\mathrm{Xs}$ which were presented on the oscilloscope face of a PDP-8/I computer. The words were generated in capital letters with the 5X7-matrix symbol generator developed at the University of Rochester [Decus No.5/8-23(B)]. The words were presented in the center of the oscilloscope and then were moved, either immediately or after one of three stationary preexposure intervals, in one of the four directions-up, down, right, or left. All five symbol strings and four movement directions were combined equally, producing three incongruent combinations for each congruent and each control condition. Four preexposure intervals of $0,100,200$, and $\mathbf{3 0 0}$ msec were combined with each Word by Direction combination to make a total of 80 different stimuli. The computer clock started with movement of the word and stopped when S's verbal response into a microphone triggered a voice key, recording the reaction times to the nearest millisecond. The verbal response also terminated the stimulus presentation, providing a form of feedback to $S$. The interval between the response to a stimulus and the next stimulus was 4 sec.

Letters were $5 \mathrm{~mm}$ high and were viewed at a distance of about $50 \mathrm{~cm} . S$ was seated in front of the computer, with the oscilloscope at approximate eye level. Movement speed was constant at $1.3 \mathrm{~cm} / \mathrm{sec}$.

Procedure. Each S was presented with eight sequences of 80 stimuli, two sequences per day for 4 successive days. Four of the sequences (mixed condition) represented the 80 possible different combinations of the five symbol strings, the four directions, and the four preexposure intervals. These "mixed" sequences were alternated with sequences of 80 stimuli that used only a single preexposure (pure condition). Each pure sequence involved four repetitions of the 20 Word by Direction combinations. The order of the four pure sequences, i.e., the four preexposure intervals, was random, with each preexposure interval appearing twice in each of the four positions (first, second, third, or last) for the eight Ss. Half of the Ss began 
with the mixed sequence and half with the pure sequence. Time between the two sequences of a session was $3 \mathrm{~min}$, which was the time for the Teletype to print out the responses of the previous first 80-trial sequence and for $E$ to load a new program on the computer.

Twenty practice trials were given to each $S$ prior to the first experimental session. Instructions were to name the direction of movement and stressed both speed and accuracy. Errors were few, but when they occurred, the stimulus combination for that trial was added automatically by the program to the end of the sequence, upon a switch closure by $E$ following the erroneous response. In addition, response latencies shorter than $250 \mathrm{msec}$ were automatically treated as errors, since they almost invariably represented an accidental activation of the voice key.

\section{Results}

Response times were analyzed in an analysis of variance with repeated measures on variables of movement direction (up, right, down, left), word (up, down, right, left, and XXXX), preexposure interval $(0,100,200$, and $300 \mathrm{msec}$ ), and a two-level factor that represented whether the sequence was pure or mixed. In addition, a factor representing the four repetitions of each stimulus combination was included. Movement direction produced a highly significant main effect, $F(3,21)=22.60, p<.001$, with the mean reaction times for up, down, right, and left being 498, 508, 562, and $563 \mathrm{msec}$, respectively. A significant main effect was also found for the variable of preexposure interval, $\mathbf{F}(3,21)=7.98, \quad \mathrm{p}<.001$. This was largely due to the long responses for the 0 preexposure ( $557 \mathrm{msec}$ ), which differed significantly (Newman Keuls-p $<.05$ ) from each of the other three means. Average response times for the 100(532 msec), 200- (521 $\mathrm{msec}$ ), and $300-\mathrm{msec}$ (524 $\mathrm{msec}$ ) preexposure intervals did not differ from each other at the .05 level of significance.

The main effect for the two-level factor corresponding to the pure vs mixed lists approached significance, $F(1,7)=4.13, .1>p>.05$, with times for the mixed lists longer than times for pure lists. The interaction of this variable with interval was significant, however, $F(3,21)=3.2, p<.05$. This resulted largely from the long response time for the 0 preexposure interval in the mixed condition (566 msec) in comparison with the pure condition (529 $\mathrm{msec}$ ). This illustrates the strong effects of the series of intervals on the responses to the short preexposure intervals. This delay occurred although little or no difference in physical appearance could be noted among the four exposure intervals.

The repetition factor also produced a significant main effect, $F(3,21)=5.4, \quad p<.01$. Means for Repetitions 1-4 were 547.1, 538.0, 524.4 , and $524.5 \mathrm{msec}$, respectively. The significant interaction of repetitions and the pure vs mixed condition, $F(3,21)=4.6, \quad p<.05$, reflects the fact that almost the total repetition effect occurred for the mixed condition, i.e., between sequences.

The word factor did not produce a significant main effect, but the interaction of words and directions was highly significant, $F(12,84)=19.08, p<.001$. This reflected the Stroop effect, i.e., the slower naming of directions when they were combined with incongruent words than when they were moving Xs. However, an even greater contribution to the interaction was made by a facilitation of direction naming when the word and direction corresponded. Means for incongruent, control, and congruent conditions were 544, 531, and $504 \mathrm{msec}$, respectively. The Newman-Keuls procedure indicated that incongruent and control conditions differed at the .05 level of significance. The congruent condition differed from the other two at the .01 level of significance. The incongruent-control condition difference occurred largely at the 0 - and 100-msec preexposures, with the mean for these two preexposures being $22 \mathrm{msec}$ compared to $5 \mathrm{msec}$ for the 200 - and 300 -msec preexposures.

\section{EXPERIMENT 2}

The findings of Experiment 1 suggest that no preexposure interval would boost interference to Stroop levels. However, the decline in interference from 100 - to $200-\mathrm{msec}$ preexposures was fairly sharp, and the possibility exists that some preexposure between 0 and $100 \mathrm{msec}$ or 100 and $200 \mathrm{msec}$ might lead to much more interference than was found for these levels themselves. To examine this possibility, a second experiment, with eight preexposures in this critical range, was carried out. Since mixing preexposures had produced artifactual increases in response time for preexposures that were short, only pure sequences were used in this experiment.

\section{Method}

Subjects. Sixteen males, who ranged in age from 17 to 24 and had recently completed Army basic training, participated in the experiment. Visual acuity in both eyes was within normal Orthorater limits.
Apparatus, materials, and procedure. The only differences between this experiment and Experiment 1 were the exclusive use of the pure condition, i.e., all stimuli in an 80-stimulus session were presented with the same stationary preexposure prior to movement and eight stationary preexposures were used: $0,31,62,86,109,133,164$, and $195 \mathrm{msec}$. The order of the eight preexposure intervals was random, with the restriction that each interval appear twice in each sequential position for the 16 Ss. One practice session of two sequences preceded the four experimental sessions of two sequences each. Sequences for a $S$ were separated by from 2 to 4 days.

\section{Results}

Data were analyzed in a repeated-measures analysis of variance with an eight-level factor of intervals, a five-level factor of words, a four-level factor of directions, and a four level factor of repetitions. Directions produced a highly significant main effect, $F(3,45)=38.2, p<.001$, with mean naming times of $436 \mathrm{msec}$ for up, $463 \mathrm{msec}$ for down, $511 \mathrm{msec}$ for right, and 505 msec for left. Intervals produced a significant main effect, $F(7,105)=3.02, p<.01$, with mean response times for the eight in order of increasing interval length being 488 , $487,489,485,472,473,471$, and 468 msec. As in Experiment 1 , words did not produce a significant main effect, but the Words by Direction interaction was highly significant, $\mathrm{F}(12,180)=36.7, \mathrm{p}<.001$. This reflects a Stroop-like interference and facilitation of naming when the movement direction and the word corresponded. To better evaluate this difference between congruent, incongruent, and control conditions, and also the interaction of these differences with the other variables, a second analysis of these data was performed where the mean of the three incongruent combinations of words with a particular movement direction was compared to the response times for the control and congruent combination for this direction. This data reduction would have the effect of reducing the variation for incongruent combinations; to compensate for this possible violation of the homogeneity of variance assumptions, only effects at the .01 level of significance or less were noted. As one would expect, highly significant main-effect differences were noted for the new-conditions variable, $F(2,30)=$ 162.2, $\mathrm{p}<.001$. The mean for the congruent combinations was $450 \mathrm{msec}$, for incongruent combinations was $490 \mathrm{msec}$, and for 
the control $\mathrm{Xs}$ the mean response time was $476 \mathrm{msec}$. All individual comparisons were significant (Newman-Keuls, $p<.01$ ). In addition, the interaction of this new factor with direction was highly significant, $F(6,90)=9.7, p<.001$. This interaction is plotted in Fig. 1. Both the facilitation of naming for congruent and delay of naming for incongruent combinations are greater for the left and right directions than for up and down. The interaction of condition with the intervals did not approach significance in this analysis, $F(14,210)=1.14$. The differences between means for the three conditions were nearly constant for all eight intervals, although a slight trend to lower interference occurred for the longest preexposures. Unlike in Experiment 1, repetitions were not significant in this analysis. This probably reflected the greater practice prior to the experimental sessions for Experiment 2.

\section{DISCUSSION}

The significant interference to naming of movement direction found in the incongruent name conditions of the present study supports predictions from two recent models of Stroop and Stroop-like interference. Morton (1969a) demonstrated that counting and other tasks are subject to interference, as color naming is in the Stroop test, and provided a model for such interference in which the critical variable is the presence of irrelevant features which readily generate name responses. Use of incongruent dimension-level names as irrelevant features in the present paradigm assures that the interference found in the present study would be predicted by Morton's model. The similarity betwe e $n$ the present movement-direction naming and the Stroop paradigm assures that the Stroop interference model of Egeth, Blecker, and Kamlet (1969) would also predict the present interference finding. The critical aspect of their formulation is that the relevant task must actively involve the cognitive apparatus. The model is less general than Morton's (1969a) and was generated to account for failures of Stroop stimuli, i.e., incongruently colored color names, to interfere with "low-level" tasks such as color matching and color counting. Color naming in their model is a "high-level" task and subject to interference, and it would be expected that the direction naming of the present study would also be interference-prone.

The applicability of these models to the present finding is of less interest perhaps than the failure of either formulation to account for the large difference in amount of interference

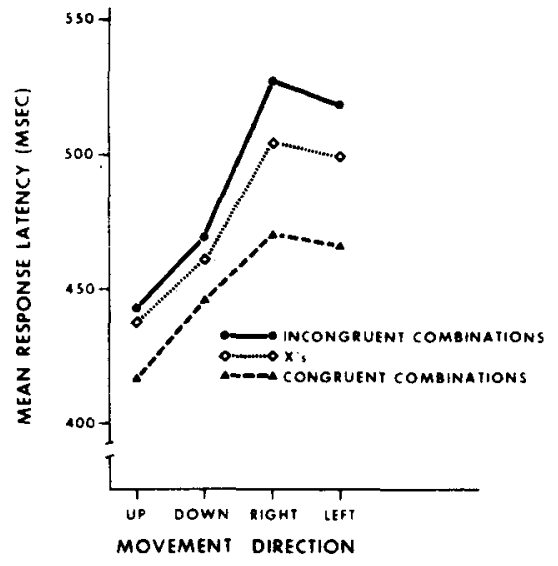

Fig. 1. Average movement-naming latency for different conditions and movement directions.

between individual stimulus versions of the regular Stroop test (Sichel \& Chandler, 1969; Dyer, 1970, 1971b) and the present direction-naming counterpart of these versions. Maximum interference in any condition of the present study was less than one-fourth of the 100 msec found for high-interference conditions of the study with preexposures of words in black prior to coloration (Dyer, 1971b). This was true despite two aspects of that study which would be expected to reduce interference compared to the present study of movement-direction naming. These were the complete feedback given in that study after each response and a longer interstimulus interval compared to that of the present work. That study of color naming provided considerably less interference than did another (Dyer, 1970) which provided no feedback of reaction times. Despite use of only three colors, the Sichel and Chandler (1969) study provided more interference than either of these other studies of color naming.

Although the interference levels of the present study differ greatly from comparable color-naming work, these results are similar to findings from other attempts to generate analogs of the Stroop test using such dimensions as arrow direction (Shor, 1970), spatial position (Shor, 1971), and achromatic shade (Dyer, 1971a). The present low interference to direction naming occurred despite a more complete integration of the direction and word aspects of the stimuli than the previous study of direction naming (Shor, 1970) in which words were written within arrows.

A possible explanation of this reduced interference to direction naming as compared to that of color naming was derived from the results of Gumenik and Glass (1970), who showed that slowing word processing relative to color processing in the Stroop test greatly reduced interference from incongruent names to color naming. Possible differences in the processing rate for movement direction compared to color may exist, with movement direction being faster. Because of this, the Gumenik and Glass conditions of fast dimension processing relative to word processing might already exist for the dimension of movement direction, even without the Gumenik and Glass manipulation of word legibility. If so, then delaying movement-direction processing relative to word processing would be expected to increase interference to direction naming to the levels that are found for color naming in the Stroop test itself. To test this hypothesis in the present study, word processing was begun prior to movement processing by presenting the word for various intervals prior to moving it. Although it was found that very long preexposures (200 and $300 \mathrm{msec}$ ) in Experiment 1 produced even less interference than no preexposure at all, no appreciable increase in interference occurred for any manipulation of the relative rates of direction and word processing. These preexposure conditions would be expected to compensate for faster peripheral processing of movement direction, and the failure of this manipulation to increase interference suggests that basic differences exist between processing of movement direction and color at higher neural levels where processed word information can interact with a processed stimulus dimension. The similar reduced interference in comparison to color which has been found in the other analogs of the Stroop test suggests that the anomalous processing occurs for the dimension of color itself.

Although speeding word processing, as was done in the present study, had little effect on the level of interference (except the reduction with long preexposures), the levels of interference for the different movement directions correlate with absolute levels of direction naming with control stimuli, as the data presented in Fig. 1 indicate. When the time to name the control stimuli is long, as it was for the left and right directions, the largest amount of interference appears for the incongruent direction-name conditions, and, conversely, when the time to name the control stimuli is short for up and down, interference is less. Thus, the hypothesis of high interference with fast word processing relative to dimension processing may still have some validity, even though 
beginning the processing of a word before movement does not provide the necessary "fast word processing relative to dimension processing." This correlation suggests that the interference locus corresponds to that stage (or those stages) of processing which provides differences in time for naming different directions when control stimuli are used. However, any generalizations from the present paradigm to color naming must be made with extreme caution, since, despite the formal similarity of the present and color-word paradigms, the low interference of the present study suggests that basic differences may exist between the two conditions. If some future manipulation of the wor $\mathrm{d} / \mathrm{movement-direction} \mathrm{stimulus}$ combination should provide interference approaching that of the color situation (as it was expected that the present stationary preexposures of the word would), analysis of these revised conditions would be expected to provide much information about the regular Stroop phenomenon.

Considerable facilitation of movement-direction naming by a congruent combination of direction name and movement direction was found in the study. This was somewhat unexpected in view of previous results (Sichel \& Chandler, 1969), although a similar result did occur in the study where color words were preexposed in black (Dyer, 1971b). The result closely resembles that of Morton (1969b), who found that card sorting was faster when numerosity and digits were congruently combined than when sorting with stimuli having only a digit or a number of nondigit symbols. The facilitation of direction naming with congruent combinations implies that considerable and perhaps total processing of the word takes place concurrently with processing of the movement direction. This eliminates one possible explanation of the interference from incongruent words to naming of movement directions: that such parallel processing of movement direction and words does not occur.

The decline in interference with the 200- and 300-msec preexposures in Experiment 1 and the trend to lower interference for the near-200-msec preexposures of Experiment 2 indicate that interference to dimension naming from words decreases with very fast as well as with very slow word processing (Gumenik \& Glass, 1970). This result for movement direction was similar to, but much less than, the decline that occurred in the recent study where color names were preexposed in black for various intervals prior to coloration (Dyer, 1971b). The difference probably reflects the large differences in interference of the two experiments. The similar finding from both studies indicates that the neural activity corresponding to viewing a word is not continuous and unchanging despite constant fixation but lasts for less than 200 msec before declining or in some other way altering its character.

The difference found between the pure and mixed conditions in Experiment 1 argues strongly for utilizing only the pure condition to obtain accurate estimates of the effect of particular preexposure intervals. The elevation of response latencies for the 0 condition appears to be related to the finding of Mowrer (1940), that simple reaction times are elevated for stimuli presented with foreperiods that differed from the average foreperiod in a sequence.

In conclusion, the results of the present study imply that the dimension of movement direction fails to provide a strong analog of the Stroop color-word test. However, this may point up important differences in the processing of different visual dimensions at higher neural levels than any one has noted previously.
REFERENCES

D ALR Y MPLE-ALFORD, E. C., \& BUDAYR, B. Examination of some aspects of the Stroop color-word test. Perceptual \& Motor Skills, 1966, 23, 1211-1214.

DYER, F. N. Word reading, color naming and Stroop interference as a function of background luminance. U.S. Army Medical Research Laboratory Report No. 889, August 17, 1970, Fort Knox, Kentuck y.

DYER, F. N. A comparison of chromatic and achromatic versions of the Stroop color-word test. Psychonomic Science, 1971a, 22, 235-237.

DYER, F. N. The duration of word meaning responses: Stroop interference for different preexposures of the word. Psych on omic Science, 1971b, 25, 229-231.

EGETH, H. E., BLECKER, D. L., \& KAMLET, A. S. Verbal interference in a perceptual comparison task. Perception \& Psychophysics, 1969, 6, 355-356.

GUMENIK, W. E, \& GLASS, R. Effects of reducing the readability of the words in the Stroop color-word test. Psychonomic Science, 1970, 20, 247-248.

JENSEN, A. R., \& ROHWER, W. D., JR. The Stroop color-word test: A review. Acta Psychologica, 1966, 25, 36-93.

MORTON, J. Categones of interference: Verbal mediation and conflict in card sorting. British Journal of Psychology, $1969 \mathrm{a}, 60,329-346$.

MORTON, $J$, The use of correlated stimulus information in card sorting. Perception \& Psychophysics, 1969b, 5, 374-376.

MOWRER, O. H. Preparatory set (expectancy): Some methods of measurement. Psychological Monographs, 1940, 52(2, Whole No. 233).

SHOR, R. E. The processing of conceptual information on spatial directions from pictorial and linguistic symbols. Acta Psychologica, 1970, 32, 346-365.

SHOR, R. E. Symbol processing speed differences and symbol interference effects in a variety of concept domains. Joumal of General Psychology, in press.

SICHEL, J. L, \& CHANDLER, K. A. The color-word interference test: The effects of varied color-word combinations upon verbal response latency. Journal of Psychology, 1969, 72, 219-231.

STROOP, J. R. Studies of interference in serial verbal reactions. Joumal of Experimental Psychology, 1935, 18, 643-662

(Accepted for publication November 19, I971.) 